\title{
BULLYING E MECANISMOS DE DESENGAJAMENTO MORAL: REVISÃO SISTEMÁTICA DA LITERATURA COM METANÁLISE
}

\author{
Wanderlei Abadio de Oliveira ${ }^{1} \mathbb{D}$; Jorge Luiz da Silva ${ }^{2} \mathbb{D}$; Eduardo Name Risk ${ }^{3} \mathbb{D}$; André Vilela Komatsu ${ }^{4} \mathbb{D}$; Marta \\ Angélica lossi Silva ${ }^{5} \mathbb{D}$; Manoel Antônio dos Santos ${ }^{5} \mathbb{D}$
}

\section{RESUMO}

O bullying é um dos principais problemas enfrentados por crianças e adolescentes nas escolas. Este estudo teve por objetivo examinar evidências disponíveis na literatura sobre o efeito do desengajamento moral no envolvimento de estudantes em situações de bullying. Para tanto, foi realizada revisão sistemática com metanálise e consulta a oito bases de dados. As publicações elegíveis foram analisadas por meio da análise conceitual e do pacote estatístico Metafor. $\mathrm{O}$ estudo foi registrado na base PROSPERO. No total, 15 artigos foram incluídos na revisão. Os dados evidenciam que o desengajamento moral é um forte preditor para o comportamento de bullying $(0 R$ 0,27; IC95\%: $0,18,0,35)$ e estudantes propensos a se desengajarem moralmente podem praticar mais bullying quando comparados com estudantes com maior engajamento moral. Conclui-se que fatores cognitivos relacionados ao desengajamento moral devem ser incluídos na discussão do fenômeno, bem como ser considerados nas propostas de intervenção antibullying.

Palavras-chave: desenvolvimento moral; violência escolar; bullying.

\section{Bullying and mechanisms of moral disengagement: systematic literature review with meta-analysis}

\section{ABSTRACT}

Bullying is one of the main problems faced by children and adolescents in schools. This study aimed to examine evidence available in the literature on the effect of moral disengagement on the involvement of students in situations of bullying. To this end, a systematic review was carried out with meta-analysis and consultation of eight databases. Eligible publications were analyzed using conceptual analysis and the Metafor statistical package. The study was registered on the PROSPERO database. In total, 15 articles were included in the review. The data show that moral disengagement is a strong predictor for bullying behavior (OR $0.27 ; 95 \% \mathrm{Cl}: 0.18,0.35)$ and students prone to morally disengage can practice more bullying when compared to students with greater moral engagement. It is concluded that cognitive factors related to moral disengagement must be included in the discussion of the phenomenon, as well as be considered in the anti-bullying intervention proposals.

Keywords: moral development; school violence; bullying.

\section{Bullying y mecanismos de no compromiso moral: revisión sistemática de la literatura con metaanálisis \\ RESUMEN}

El bullying es uno de los principales problemas enfrentados por niños y adolescentes en las escuelas. En este estudio se tuvo por objetivo examinar evidencias disponibles en la literatura sobre el efecto del no compromiso moral en el envolvimiento de estudiantes en situaciones de bullying. Para tanto, se realizó revisión sistemática con metaanálisis y consulta a ocho bases de datos. Las publicaciones elegibles se analizaron por intermedio del análisis conceptual y del paquete estadístico Metafor. El estudio fue registrado en la base PROSPERO. En total, 15 artículos se incluyeron en la revisión. Los datos evidencian que el no compromiso moral es un fuerte predictor para el comportamiento de bullying (OR 0.27; IC95\%: 0.18, 0.35) y estudiantes propensos a no comprometerse moralmente pueden practicar más bullying cuando comparados con estudiantes con más compromiso moral. Se concluye que factores cognitivos relacionados a la falta de compromiso moral deben ser incluidos en la discusión del fenómeno, así como ser considerados en las propuestas de intervención antibullying.

Palabras clave: desarrollo moral; violencia escolar; bullying.

\footnotetext{
${ }^{1}$ Pontifícia Universidade Católica de Campinas - Campinas - São Paulo - SP - Brasil; wanderleio@hotmail.com

2 Universidade de Franca - Franca - São Paulo - SP - Brasil; jorge.silva@unifran.edu.br

${ }^{3}$ Universidade Federal de São Carlos - São Carlos - São Paulo - SP - Brasil; eduardonrisk@gmail.com

${ }^{4}$ Universidade de São Paulo - São Paulo - São Paulo - SP - Brasil; andevk@gmail.com

${ }^{5}$ Universidade de São Paulo - Ribeirão Preto - São Paulo - SP - Brasil; maiossi@eerp.usp.br; masantos@ffclrp.usp.br
} 


\section{INTRODUÇÃO}

A ocorrência de bullying repercute negativamente no desenvolvimento, na saúde e no processo ensinoaprendizagem de crianças e adolescentes (Crochik, 2016; Fernandes, Yunes, \& Finkler, 2020; Zequinão, Medeiros, Silva, Pereira, \& Cardoso, 2020). O fenômeno corresponde a um tipo de violência caracterizado pela intencionalidade dos agressores em infligir dor ou sofrimento às vítimas, pela reiteração dos comportamentos agressivos e pelo desequilíbrio de poder entre os estudantes envolvidos (Olweus, 2013). Trata-se de fenômeno de grupo, pois cada participante apresenta tendência para reverberar ou manter as regras e condições estabelecidas pelos integrantes com maior status ou popularidade (Sijtsema et al., 2014).

Embora fenômeno de grupo, as questões individuais devem ser consideradas na análise de sua dinâmica. Esse aspecto foi valorizado e assinalado desde os primeiros estudos sobre a temática conduzidos por Dan Olweus desde a década de 1970 (Olweus, 2013). É essencial compreender quais são os mecanismos individuais subjacentes ao comportamento de bullying, por meio de pesquisas, de modo a fornecer subsídios para programas de prevenção e intervenção nas escolas (Sijtsema et al., 2014). Os fatores cognitivos e outras dimensões do desenvolvimento são incluídos nessa discussão sobre os fatores individuais e podem contribuir para a ocorrência do bullying.

Assim, com relação aos fatores individuais, o desengajamento moral se refere a uma série de processos cognitivos considerados válidos para elucidar o comportamento agressivo ou desviante de crianças e adolescentes (Bandura et al., 1996). Segundo o autor, os processos do desengajamento moral correspondem a mecanismos de autojustificação cognitiva para ações transgressivas que foram hipoteticamente aprendidas e socializadas em contextos sociais específicos. Tal constructo teórico foi elaborado em consonância com pressupostos da Teoria da Aprendizagem Social e da Psicologia Social Cognitiva.

O desengajamento moral é composto por oito mecanismos: (1) justificativa moral, (2) comparação vantajosa, (3) linguagem eufemística, (4) deslocamento de responsabilidade, (5) difusão de responsabilidade, (6) distorção de consequências, (7) desumanização e (8) culpabilização da vítima (Bandura et al., 1996). Os três primeiros mecanismos se referem à reestruturação cognitiva, com base na qual as pessoas percebem o comportamento negativo ou imoral de forma mais positiva (justificativa moral) ou interpretam que tal comportamento é menos imoral/danoso quando comparado com outro (comparação vantajosa). Em outras situações, as pessoas podem usar a linguagem como recurso para minimizar ou obscurecer o impacto negativo do comportamento (linguagem eufemística)
(Bandura et al., 1996; Hymel \& Bonanno, 2014).

Os dois mecanismos seguintes, deslocamento e difusão de responsabilidade, permitem minimizar o papel de agente do comportamento imoral ou negativo, deslocando a responsabilidade pessoal para outras pessoas ou para o grupo de pares. A distorção de consequências envolve desvirtuar ou desconsiderar as consequências do comportamento percebido como mais positivo. Já os mecanismos desumanização e culpabilização permitem considerar a vítima como merecedora dos comportamentos imorais ou negativos, bem como indigna de receber tratamentos dispensados ao ser humano em si (Bandura et al., 1996; Hymel \& Bonanno, 2014).

Esses oito mecanismos são considerados como de autorregulação e criam nexo entre cognição, emoção moral e comportamento engajado ou desengajado moralmente (Bandura et al., 1996; Hymel \& Bonanno, 2014). Segundo a literatura científica, os comportamentos agressivos e a prática do bullying entre estudantes têm sido associados ao desengajamento moral, pois os agressores, via de regra, justificam racionalmente suas ações, não apresentam sentimento de culpa e não reconhecem os danos causados às vítimas (Bandura et al., 1996; Sijtsema et al., 2014). Assim, este estudo teve por objetivo examinar as evidências disponíveis na literatura sobre o efeito do desengajamento moral no envolvimento de estudantes em situações de bullying.

\section{MÉTODO}

\section{Tipo de Estudo}

O método de revisão sistemática de literatura com metanálise foi empregado para identificar, avaliar e interpretar dados de estudos realizados sobre a interface entre bullying e desengajamento moral. O seguinte processo de execução foi adotado: (1) formulação da questão norteadora, (2) seleção das bases de dados, (3) definição da estratégia de busca, (4) procedimento de coleta e seleção de dados, (5) análise e apresentação dos resultados e (6) apresentação da revisão sistemática (Carvalho, Pianowski, \& Santos, 2019). O protocolo para realização dessa revisão sistemática foi registrado na base PROSPERO (International Prospective Register of Systematic Reviews) sob o número: CRD42018099782.

\section{Formulação da Questão Norteadora}

Para a definição da questão de pesquisa, foi aplicado o modelo PVO, acrônimo formado por P - participantes/ contexto/fenômeno estudado, $V$ - variáveis em estudo e O - desfecho/resultados. Assim, a pergunta norteadora desse estudo foi: Qual a relação entre os mecanismos de desengajamento moral e o envolvimento de estudantes em situações de bullying escolar? O modelo 
PVO foi estruturado da seguinte forma: $P$ - estudantes envolvidos em situações de bullying, $\mathrm{V}$ - bullying e desengajamento moral, $\mathrm{O}$ - identificar, descrever e analisar resultados sobre a relação entre as variáveis escolhidas.

\section{Seleção das Bases de Dados}

A busca contemplou sete fontes de informação, que indexam a literatura das seguintes áreas: Psicologia, Saúde, Educação e Multidisciplinar. Foram consultadas as bases de dados Web of Science, PsycINFO, Scopus, PubMed, SciELO, PePSIC e LILACS.

\section{Definição da Estratégia de Busca}

Foram utilizados os descritores bullying e desengajamento moral (bullying and moral disengagement) em todas as bases consultadas. Os critérios de inclusão foram: (1) estudos com delineamentos transversais ou longitudinais, (2) estudos com avaliação de prevalência de bullying e desengajamento moral, (3) estudos com crianças e adolescentes em idade escolar e (4) textos publicados em inglês, espanhol ou português no período de janeiro de 2013 a agosto de 2018. Foram excluídos estudos que não abordassem ou que referissem apenas secundariamente os temas em foco (desengajamento moral e bullying), bem como obras no formato de livro, capítulo de livro, tese, dissertação, editorial, cartas ao editor e anais de evento científico. Nesta revisão não foram incluídos estudos sobre cyberbullying, pois a literatura especializada considera esse fenômeno como relacionado, porém distinto em termos de características e dinâmica quando comparado ao bullying tradicional.

\section{Procedimentos de Coleta e Seleção de Dados}

A busca dos artigos nas bases de dados ocorreu no mês de setembro de 2018 e foi realizada por dois revisores independentes. Considerando as diferentes formas de apresentação da autoria dos artigos nas bases, as duplicatas foram eliminadas via software de gerenciamento de referências EndNote e manualmente no Excel. A primeira triagem foi realizada pelos dois pesquisadores por meio da leitura de títulos e resumos identificados no cruzamento dos descritores utilizados. No segundo momento, os pesquisadores recuperaram e leram na íntegra os artigos selecionados. Divergências entre ambos foram discutidas até a obtenção de consenso.

\section{Análise e Apresentação dos Resultados}

$\mathrm{O}$ armazenamento e gerenciamento dos dados dos artigos foram realizados em planilha Exce/ contemplando os seguintes indicadores: título, autores, ano e periódico, país onde o estudo foi desenvolvido, tipo de estudo, número de participantes, instrumento utilizado para mensurar episódios de bullying, instrumento utilizado para mensurar o desengajamento moral, objetivos do estudo, principais resultados e limitações apresentadas. Essa sistematização facilitou as análises descritivas e a apreciação crítica dos estudos selecionados. Também foi desenvolvida análise conceitual (Walker \& Avant, 2018) para identificar como o constructo desengajamento moral está sendo delineado em estudos sobre bullying.

No processo de metanálise o efeito de medida utilizado para a análise foi o coeficiente $r$ de Pearson entre os escores obtidos para bullying e desengajamento moral nos estudos revisados. As análises foram realizadas com o pacote estatístico Metafor (Viechtbauer, 2010) do software R, versão 3.5.1. A avaliação da heterogeneidade entre os estudos e sua magnitude foi estimada mediante os testes $Q$ de Cochran e o índice $l^{2}$. As porcentagens de referência para a heterogeneidade, considerando-se o índice $l^{2}$, foram: próximas de $25 \%$ (baixa), próximas de $50 \%$ (moderada) e próximas de $75 \%$ (alta) (Higgins \& Thompson, 2002). Considerou-se o nível de significância de $5 \%$.

Os artigos que compõem essa revisão também foram avaliados por dois pesquisadores independentes quanto à qualidade metodológica, a partir de dois instrumentos: (1) Prevalence Critical Appraisal Instrument e (2) Appraisal tool for CrossSectional Studies (AXIS tool). O Prevalence Critical Appraisal Instrument contempla 10 questões para avaliar a qualidade metodológica de estudos que apresentam dados de prevalência (Munn et al., 2014). O AXIS tool é um instrumento construído para subsidiar decisões de intervenção por meio da avaliação da qualidade e do risco de viés de pesquisas transversais divulgadas na literatura científica (Downes et al., 2016). Considerando a questão norteadora da pesquisa, o AXIS tool foi adaptado para avaliar também os cinco artigos com delineamento longitudinal.

\section{RESULTADOS}

\section{Análise Descritiva}

Na primeira etapa do estudo todos os resultados foram considerados elegíveis e totalizaram 27 artigos, eliminadas as repetições entre os cruzamentos ou entre as bases de dados. $\mathrm{O}$ fluxo do processo de construção do corpus do estudo consta na Figura 1.

A análise descritiva dos artigos incluídos na revisão permitiu identificar a distribuição das publicações por ano ao longo do período estudado (2013-2018). Não foram verificadas tendências à concentração, pois os estudos se distribuíram de forma homogênea ao longo dos anos. Os artigos foram publicados em 11 periódicos, $47 \%$ foram publicados em periódicos com alto nível de especialidade (Journal of School Violence, Journal of Interpersonal Violence e Aggressive Behavior). A respeito do país de origem dos estudos revisados, a Itália, seguida por Austrália, Grécia e Suécia foram os 


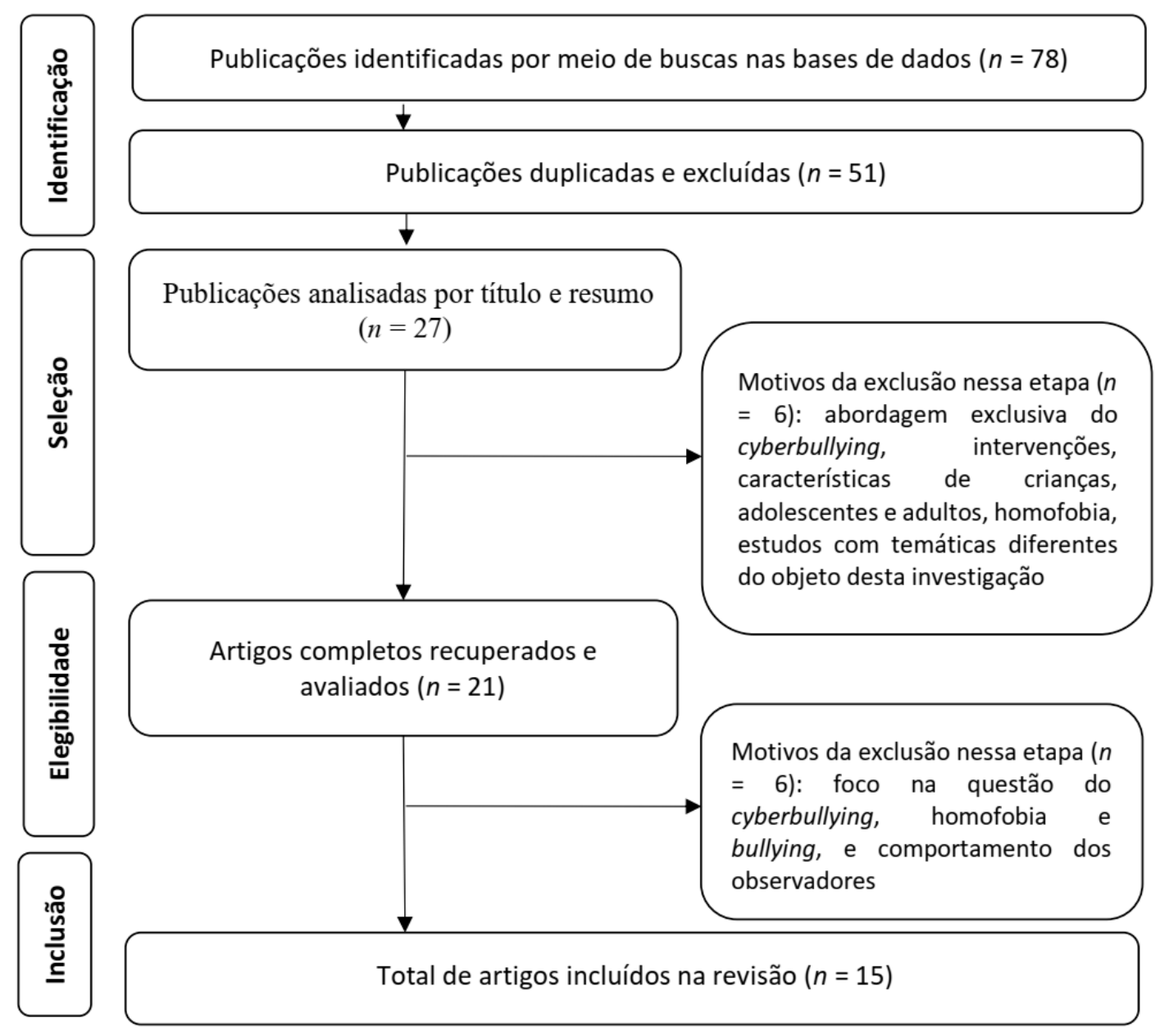

Figura 1. Fluxograma PRISMA do Processo de Busca e Seleção dos Artigos.

Fonte: elaborado pelos autores.

contextos de maior desenvolvimento de pesquisas sobre bullying e desengajamento moral. Não foram encontrados estudos desenvolvidos no Brasil.

Do total de estudos incluídos, 11 apresentavam desenho transversal e utilizaram amostras de participantes robustas, ou seja, com número total que permite generalizações. Todos os artigos reportavam resultados quantitativos. Foi expressivo o número de pesquisas que adotou o Olweus Bully/Victim Questionnaire ou adaptações desse questionário. No que se refere à avaliação de desengajamento moral, a maioria dos estudos utilizou o Moral Disengagement Scale (Bandura et al., 1996).

Em geral, as formas de manifestação do bullying foram significativamente correlacionadas positivamente com desengajamento moral, assim como os meninos referiram mais desengajamento moral e envolvimento em situações de bullying quando comparados às meninas (Kokkinos \& Kipritsi, 2018; Sijtsema et al., 2014; Thornberg et al., 2015; Van Noorden et al., 2014; Wang,
Ryoo et al., 2017). Estudantes que relataram praticar bullying também apresentaram tendência a justificar seus comportamentos por meio dos mecanismos que compõem o constructo desengajamento moral anteriormente apresentado (Orue \& Calvete, 2016; Thornberg \& Jungert, 2014)

Nesse cenário, estudantes com maiores pontuações em escalas de desengajamento moral apresentaram como resultado relacionado o aumento na prática do bullying contra os colegas (Obermann, 2013; Wang, Ryoo et al., 2017; Wang, Yang et al., 2017). Além disso, na dinâmica do bullying os meninos apresentaram menores níveis de culpa ao agredirem os colegas (Fitzpatrick \& Bussey, 2017; Mazzone et al., 2016; Menesini et al., 2015).

\section{Análise Conceitual}

A partir da análise conceitual foi possível, primeiramente, identificar os usos ou definições de desengajamento moral empregados nos estudos 
selecionados. Houve consenso e o constructo em questão foi definido como uma série de processos ou mecanismos cognitivos utilizados para aliviar a culpa e permitir a adoção de comportamentos antissociais ou agressivos. Os dados relativos a nível de análise estão representados na Figura 2.

O modelo apresentado ressalta a integração teórica entre aspectos cognitivos e emocionais enquanto duas dimensões morais que contribuem para explicar diferenças individuais que são relevantes nos comportamentos de bullying (Thornberg et al., 2015). A propensão dos estudantes ao desengajamento moral foi associada ao aumento do bullying, considerado um fenômeno de grupo (Fitzpatrick \& Bussey, 2017). Além disso, foi possível verificar que o desengajamento moral é um preditor significativo para o comportamento de bullying (Travlos et al., 2018). Por outro lado, os conceitos relacionados ao desengajamento moral podem ser interpretados como respostas que auxiliariam no combate ao bullying, sendo úteis em intervenções que focalizem a clarificação e análise de valores, bem como o desenvolvimento de empatia, solidariedade e altruísmo (Kokkinos \& Kipritsi, 2018; Wang, Ryoo et al., 2017).

\section{Meta-análise e Avaliação de Qualidade Metodológica}

Oito estudos preencheram os critérios exigidos (escores em bullying e desengajamento moral) para o

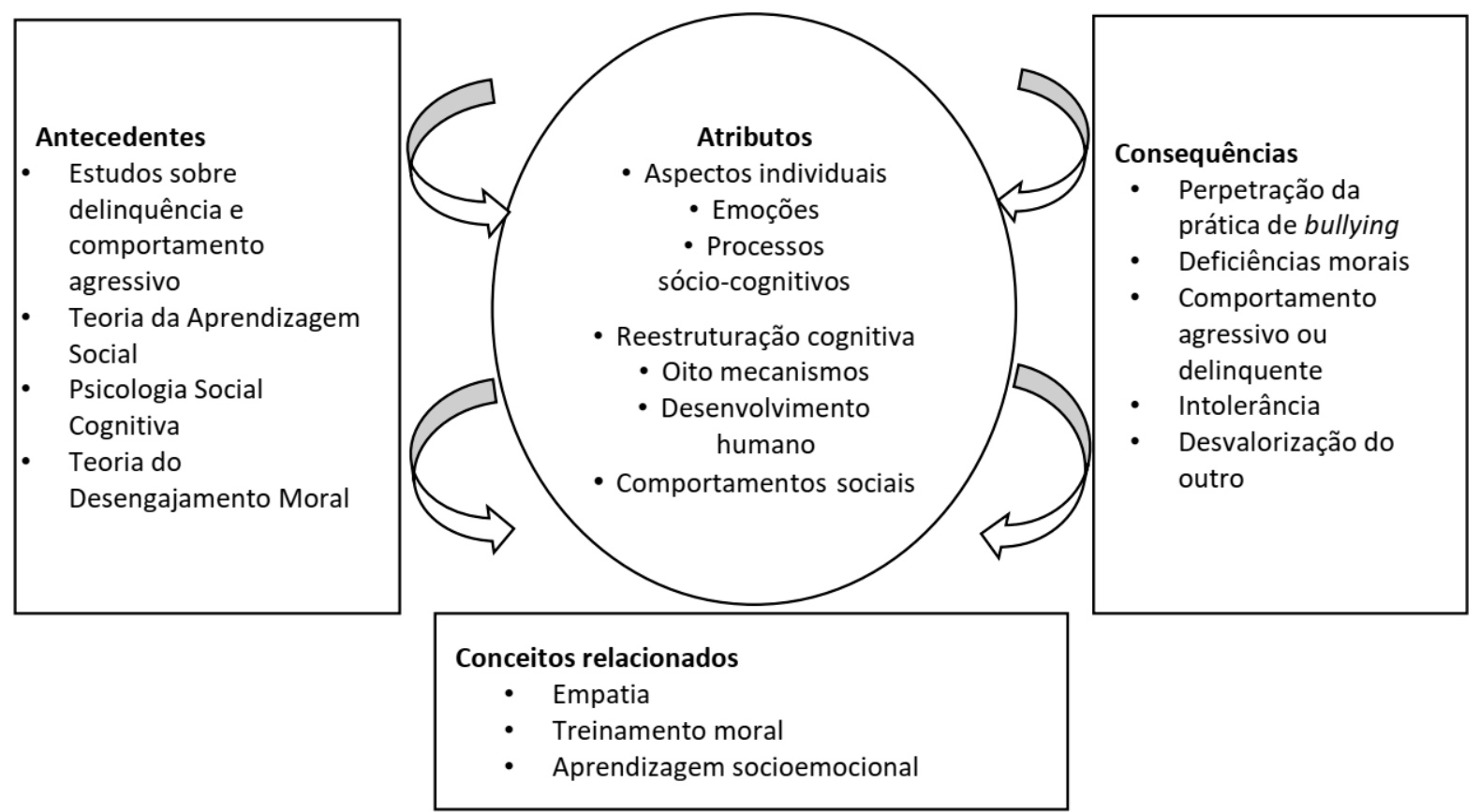

Figura 2. Estrutura Conceitual de Desengajamento Moral em Estudos sobre Bullying Escolar. Fonte: elaborado pelos autores.

desenvolvimento da metanálise. Em um dos estudos (Travlos et al., 2018) essa medida não estava disponível, sendo obtida por meio da transformação do valor de $F$ para valor $Z$ e depois para o valor de $r$ conforme procedimento descrito na literatura (Hughett, 2007). Os resultados da metanálise estão na Figura 3.

Os resultados apresentados demonstram efeitos estatisticamente significantes sobre a relação entre bullying e desengajamento moral com diferença média combinada: 0.27 (IC95\%: 0.18, 0.35). Houve heterogeneidade entre os estudos, com alta variabilidade ( $12=92.7 \%$ ). Assim, no conjunto de dados revisados, o desengajamento moral foi significativamente associado com adoção de comportamentos de bullying.

No que tange à qualidade metodológica dos estudos revisados, aferida com base na aplicação do instrumento Prevalence Critical Appraisal Instrument, sete estudos foram classificados como de nível A. O mesmo ocorreu na avaliação do risco de viés e qualidade segundo os itens de avaliação do instrumento AXIS tool. Os estudos avaliados como de nível $B$ ou de qualidade moderada apresentaram fragilidade nos procedimentos de definição de amostra em relação à população, nos cuidados com os participantes não respondentes e na determinação dos critérios para estabelecer a significância estatística. $\mathrm{O}$ artigo avaliado como de alto risco de viés e baixa qualidade metodológica (Kollerová et al., 2014) apresentou ainda limitações na descrição detalhada dos procedimentos de coleta de dados. Os resultados da avaliação da qualidade metodológica dos 


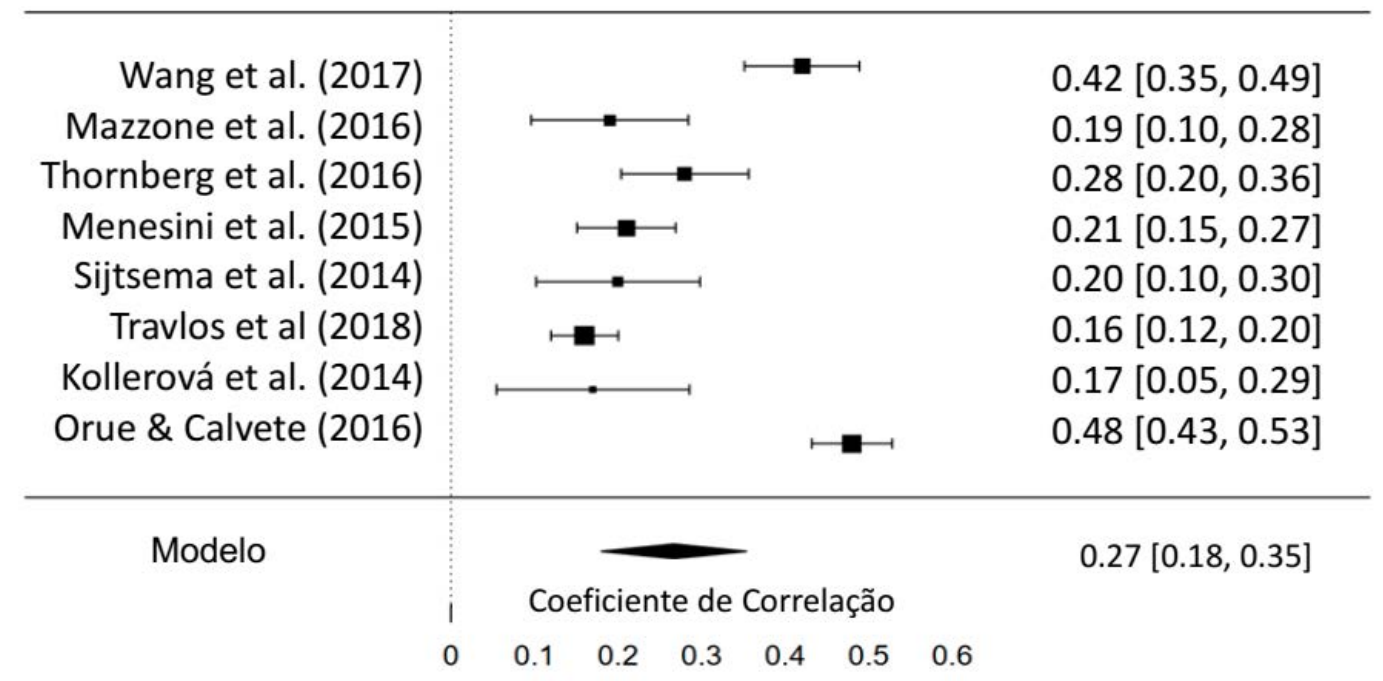

Figura 3. Resultados da Metanálise sobre Desengajamento Moral em Estudos sobre Bullying Escolar.

Nota: Forest plot do resultado da metanálise da correlação entre bullying e desengajamento moral. Heterogeneidade: $Q=131,4$, $d f=8,0,(p<0,001), l^{2}=92,7 \%$. Teste para efeito global: $Z=6(p<0,001)$. Fonte: elaborado pelos autores.

artigos estão resumidos na Quadro 1.

Também foram sumarizadas as quatro principais limitações referidas nos estudos revisados, conforme pressupõem as orientações para a construção de revisões sistemáticas (Carvalho et al., 2019). Primeiramente, a principal limitação apresentada se refere à natureza transversal da maioria dos estudos $(n=11)$, pois esse delineamento impede interpretações causais a partir dos resultados (Kokkinos \& Kipritsi, 2018; Mazzone et al., 2016; Menesini et al., 2015). Em segundo lugar, o tamanho e as características das amostras estudadas foram apontados como forte limitação em dois casos específicos (Orue \& Calvete, 2016; Robson \& Witenberg, 2013). A terceira limitação se refere ao uso de instrumentos do tipo autorrelato, que tendem a aumentar a ocorrência do fenômeno denominado desejabilidade social (Thornberg \& Jungert, 2014; Wang, Ryoo et al., 2017).

\section{DISCUSSÃO}

Os resultados revisados confirmaram que o desengajamento moral é um forte preditor para o comportamento de bullying, não apenas enquanto influência direta, mas também como influência indireta por meio da ausência dos sentimentos de empatia e de culpa, inversos ao desengajamento moral. Sumariamente, os dados sugerem que estudantes propensos ao desengajamento moral podem praticar mais bullying quando comparados a estudantes com maior engajamento moral. Os meninos se desengajavam moralmente mais do que as meninas, assim como praticavam mais bullying.
A maior parte dos estudos é de natureza transversal e confirmou a existência de forte relação entre desengajamento moral e bullying. Esses dados foram consistentes com os estudos longitudinais incluídos na revisão e também são respaldados pela literatura clássica da área (Bandura et al., 1996). Entrementes, no que se refere à prevalência de desengajamento moral e comportamentos de bullying entre os meninos, esses dados não são considerados conclusivos, sobretudo naqueles oriundos de estudos transversais (Wang, Ryoo, et al., 2017). Essa constatação indica necessidade de investigações futuras específicas sobre a relação entre três variáveis: gênero, bullying e desengajamento moral.

No que concerne à localização das pesquisas revisadas, observou-se que elas ficaram circunscritas aos países considerados como desenvolvidos. Esse aspecto indica que países em desenvolvimento, como o Brasil ou outros da América Latina, devem investir na investigação de outras dimensões, como os processos cognitivos ou as interações familiares que também influenciam na dinâmica do bullying.

A perspectiva adotada a respeito do conceito de desengajamento moral nos estudos sobre bullying ampliou a compreensão sobre esse problema. Conforme abordagem holística e funcional, os antecedentes, os atributos, as consequências e os conceitos relacionados a esse processo cognitivo estimularam atenção para outras dimensões da agressão entre pares e seus fatores ambientais interativos ou individuais associados, como o desenvolvimento moral (Bandura et al., 1996). Os estudantes que adotam comportamentos de bullying, em geral, apresentam dificuldades nesse sentido - 


\begin{tabular}{|c|c|c|c|c|c|c|}
\hline \multirow[t]{2}{*}{ Referências } & \multicolumn{3}{|c|}{$\begin{array}{l}\text { Nível de qualidade } \\
\text { metodológica }\end{array}$} & \multicolumn{3}{|c|}{ Qualidade metodológica } \\
\hline & A & B & $\mathrm{C}$ & Alta & Moderada & Baixa \\
\hline Koffinos e Kipritsi, 2018 & & $\checkmark$ & & & $\checkmark$ & \\
\hline Wang et al., 2017b & & $\checkmark$ & & & $\checkmark$ & \\
\hline Wang et al., 2017a & $\checkmark$ & & & & $\checkmark$ & \\
\hline Mazzone et al., 2016 & & $\checkmark$ & & & $\checkmark$ & \\
\hline Thornberg et al., 2016 & $\checkmark$ & & & & $\checkmark$ & \\
\hline Menesini et al., 2015 & $\checkmark$ & & & $\checkmark$ & & \\
\hline Sijtsema et al., 2014 & $\checkmark$ & & & & $\checkmark$ & \\
\hline Van Noorden et al., 2014 & & $\checkmark$ & & & $\checkmark$ & \\
\hline Thornberg e Jungert, 2014 & $\checkmark$ & & & & $\checkmark$ & \\
\hline Obermann, 2013 & & $\checkmark$ & & $\checkmark$ & & \\
\hline Robson e Witenberg, 2013 & & $\checkmark$ & & $\checkmark$ & & \\
\hline Travlos et al., 2018 & & $\checkmark$ & & & $\checkmark$ & \\
\hline Fitzpatrick e Bussey, 2017 & $\checkmark$ & & & $\checkmark$ & & \\
\hline Kollerová et al., 2014 & & & $\checkmark$ & & $\checkmark$ & \\
\hline Orue e Calvete, 2016 & $\checkmark$ & & & $\checkmark$ & & \\
\hline
\end{tabular}

Quadro 1 Nível de Qualidade Metodológica dos Estudos Revisados Segundo os Critérios: Representatividade e Tamanho Amostral, Tipo de Recrutamento, Apresentação da Temática e Desenho, Análise de Dados e Consistência Interna.

intolerância e pouca habilidade para resolver conflitos de forma assertiva (Menesini et al., 2015).

A variável desengajamento moral abordada nos estudos sobre bullying refere-se à vulnerabilidade individual de duplo significado. Primeiro, trata-se de funcionamento cognitivo e emocional flexível frente à ruptura de regras e normas de convívio social (Menesini et al., 2015; Thornberg \& Jungert, 2014; Thornberg et al., 2015). Segundo, a ausência de culpa perpetua comportamentos agressivos (Mazzone et al., 2016; Obermann, 2013; Wang, Ryoo et al., 2017). Por outro lado, a empatia, enquanto capacidade de resposta ao sofrimento do outro, é um conceito importante e relacionado ao desengajamento moral (Hymel \& Bonanno, 2014).

Um ensaio teórico considerou que a teoria do desengajamento moral fornece sistema conceitual para a compreensão do comportamento de bullying entre crianças e adolescentes, principalmente pela característica dos agressores de não apresentarem sentimentos de autocondenação (Hymel \& Bonanno, 2014). Nesse sentido, infere-se que cognição e emoções morais podem explicar comportamentos negativos ou positivos a partir das diferenças individuais (Thornberg et al., 2015). Assim, o desengajamento moral explicaria, em alguma medida, os comportamentos de bullying.

Essa compreensão é ampliada, pois a falta de conteúdo moral em crianças e adolescentes em idade escolar indica que esses indivíduos não enxergam os colegas como dignos de valor ou de existência humana em si (Obermann, 2013; Wang, Ryoo, et al., 2017). Teoricamente, esse processo pode ser explicitado por diferentes mecanismos e questões sociais/psicossociais importantes para compreender o engajamento moral como sistêmico e não apenas como pertencente ao campo individual. Mesmo em face dos componentes relacionados à singularidade do indivíduo, não se pode ignorar as relações interpessoais promotoras de 
processos de internalização das regras sociais e dos modos como as interações sociais acontecerão ao longo do ciclo vital (Lambe et al., 2017).

Entretanto, mesmo diante desses resultados e de suas implicações, os estudos revisados apresentam lacunas relacionadas à diversidade de informações ou à maneira como essas foram coletadas. Ao elencar as principais limitações dos estudos revisados pretende-se incentivar novas pesquisas que se dediquem a esclarecer as variáveis analisadas. Essas recomendações visam a impedir que outros pesquisadores e profissionais sigam rumos inadequados no desenvolvimento de agendas de práticas ou pesquisas. Em paralelo a essa dimensão, os instrumentos utilizados nos processos de coleta de dados não foram capazes de mensurar percepções ou aspectos sensivelmente subjetivos, condição que poderia ser minimizada a partir do desenvolvimento de pesquisas qualitativas, por exemplo.

Em relação à análise da qualidade metodológica dos estudos revisados, identificou-se certa heterogeneidade, pois a qualidade das evidências foi avaliada como moderada na maioria dos estudos, não sendo detectada qualquer imprecisão grave nesse nível de análise. Esse resultado permite inferir que as evidências revisadas são válidas, mas que ainda são requeridos estudos adicionais, com diferentes delineamentos e tamanhos amostrais diferenciados, para que as limitações apontadas possam ser amenizadas ou suplantadas. Essas observações são sugeridas pelos autores das pesquisas, que via de regra não são consideradas conclusivas.

A partir dos resultados revisados, verifica-se que os programas de intervenção destinados a reduzir o bullying nas escolas devem também se concentrar nas questões individuais e estimular relações empáticas baseadas em comportamentos positivos do tipo ajuda e valorização positiva do outro, solidariedade e engajamento moral (Sijtsema et al., 2014; Wang, Ryoo, et al., 2017). Como os meninos foram identificados como os que mais apresentaram comportamentos de desengajamento moral e de bullying (Kokkinos \& Kipritsi, 2018; Wang, Ryoo, et al., 2017a), sugere-se que esses programas se concentrem nesse grupo. Essas iniciativas poderão favorecer o desenvolvimento de comportamentos pró-sociais, caracterizados pelo altruísmo e pela cidadania, e impactar positivamente no desempenho escolar dos estudantes.

\section{CONSIDERAÇÕES FINAIS}

O ponto forte desse estudo reside na sistematização inédita de evidências sobre a relação entre as variáveis desengajamento moral e bullying escolar. Todos os resultados analisados indicaram que o desengajamento moral aumenta o risco dos estudantes se envolverem em situações de bullying. Contudo, esses achados devem ser interpretados à luz de suas três principais limitações. Primeiro, a análise restringe-se aos conceitos publicados sobre desengajamento moral e bullying e ignora variáveis que eventualmente poderiam ser predominantes em alguns dos estudos revisados (maus tratos e empatia, por exemplo). Em segundo lugar, a estratégia de busca com ênfase nos termos desengajamento moral e bullying pode ter omitido contribuições científicas que não declararam explicitamente essas variáveis em seus títulos ou resumos.

Por fim, destaca-se que o presente estudo oferece implicações práticas e de pesquisa. Em termos práticos, aspectos relacionados à empatia e tolerância à diversidade devem ser incluídos na pauta dos programas de intervenção antibullying. Ao mesmo tempo, estudantes que praticam bullying devem participar de iniciativas que apontem os efeitos negativos de seus comportamentos e como os conflitos podem ser resolvidos de forma não-violenta. Para estudos futuros é imperativo o uso de diferentes modelos de pesquisa (exploratórias, longitudinais e qualitativas, por exemplo) a fim de abordar as relações entre o constructo desengajamento e a ocorrência do bullying.

\section{REFERÊNCIAS}

Bandura, A.; Barbaranelli, C.; Caprara, G. V.; Pastorelli, C. (1996). Mechanisms of moral disengagement in the exercise of moral agency. Washington: American Psychological Association.

Carvalho, L. F., Pianowski, G., \& Santos, M. A. (2019). Guidelines for conducting and publishing systematic reviews in Psychology. Estudos de Psicologia (Campinas), 36, e180144. doi:10.1590/1982-0275201936e180144

Crochík, J. L. (2016). Hierarchy, violence and bullying among students of public middle schools. Paidéia (Ribeirão Preto), 26(65), 307-315. doi:10.1590/1982-43272665201608

Downes, M. J.; Brennan, M. L.; Williams, H. C.; Dean, R. S. (2016). Development of a critical appraisal tool to assess the quality of cross-sectional studies (AXIS). BMJ Open, 6(12), e011458. doi:10.1136/bmjopen-2016-011458

Fitzpatrick, S.; Bussey, K. (2017). The role of moral disengagement on social bullying in dyadic very best friendships. Journal of School Violence, 17(4), 417-429. do i:10.1080/15388220.2017.1355810

Fernandes, G., Yunes, M. A. M., \& Finkler, L. (2020). The social networks of adolescent victims of domestic violence and bullying. Paidéia (Ribeirão Preto), 30, e3007. doi:10.1590/1982-4327e3007

Higgins, J. P. T.; Thompson, S. G. (2002). Quantifying heterogeneity in a meta-analysis. Statistics in Medicine, 21(11), 1539-1558. doi:10.1002/sim.1186

Hughett, P. (2007). Accurate computation of the f-to-z and t-to-z transforms for large arguments. Journal of Statistical Software, 1(1), 1-5. 
Hymel, S.; Bonanno, R. A. (2014). Moral disengagement processes in bullying. Theory into Practice, 53(4), 278-285. doi:10.1080/00405841.2014.947219

Kokkinos, C. M.; Kipritsi, E. (2018). Bullying, moral disengagement and empathy: Exploring the links among early adolescents. Educational Psychology, 38(4), 535-552. doi:10.1080/01443410.2017.1363376

Kollerová, L.; Janošová, P.; Říčan, P. (2014). Moral disengagement from bullying: The effects of gender and classroom. New Educational Review, 37(3), 280-291.

Lambe, L. J.; Hudson, C. C.; Craig, W. M.; Pepler, D. J. (2017). Does defending come with a cost? Examining the psychosocial correlates of defending behaviour among bystanders of bullying in a Canadian sample. Child Abuse \& Neglect, 65, 112-123. doi:10.1016/j.chiabu.2017.01.012

Mazzone, A.; Camodeca, M.; Salmivalli, C. (2016). Interactive effects of guilt and moral disengagement on bullying, defending and outsider behavior. Journal of Moral Education, 45(4), 419-432. doi:10.1080/03057240.2016 .1216399

Menesini, E.; Palladino, B. E.; Nocentini, A. (2015). Emotions of moral disengagement, class norms, and bullying in adolescence: A multilevel approach. Merrill-Palmer Quarterly-Journal of Developmental Psychology, 61(1), 124-143. doi:10.13110/merrpalmquar1982.61.1.0124

Munn, Z.; Moola, S.; Riitano, D.; Lisy, K. (2014). The development of a critical appraisal tool for use in systematic reviews addressing questions of prevalence. International Journal of Health Policy and Management, 3(3), 123-128. doi:10.15171/ijhpm.2014.71

Obermann, M.-L. (2013). Temporal aspects of moral disengagement in school bullying: Crystallization or escalation? Journal of School Violence, 12(2), 193-210. do i:10.1080/15388220.2013.766133

Olweus, D. (2013). School bullying: Development and some important challenges. Annual Review of Clinical Psychology, 9(1), 751-780. doi:10.1146/annurevclinpsy-050212-185516

Orue, I.; Calvete, E. (2016). Psychopathic traits and moral disengagement interact to predict bullying and cyberbullying among adolescents. Journal of Interpersonal Violence, 34(11), 2313-2332. doi:10.1177/0886260516660302

Robson, C.; Witenberg, R. T. (2013). The influence of moral disengagement, morally based self-esteem, age, and gender on traditional bullying and cyberbullying. Journal of School Violence, 12(2), 211-231. doi:10.1080/153882 20.2012.762921

Sijtsema, J. J.; Rambaran, J. A.; Caravita, S. C. S.; Gini, G. (2014). Friendship selection and influence in bullying and defending: Effects of moral disengagement. Developmental Psychology, 50(8), 2093-2104. doi:10.1037/a0037145

Thornberg, R.; Jungert, T. (2014). School bullying and the mechanisms of moral disengagement. Aggressive Behavior, 40(2), 99-108. doi:10.1002/ab.21509

Thornberg, R.; Pozzoli, T.; Gini, G.; Jungert, T. (2015). Unique and interactive effects of moral emotions and moral disengagement on bullying and defending among school children. Elementary School Journal, 116(2), 322-337. doi:10.1086/683985

Travlos, A. K.; Tsorbatzoudis, H.; Barkoukis, V.; Douma, I. (2018). The effect of moral disengagement on bullying: Testing the moderating role of personal and social factors. Journal of Interpersonal Violence, 36(5-6), 2262-2281. doi:10.1177/0886260518760012

Van Noorden, T. H. J.; Haselager, G. J. T.; Cillessen, A. H. N.; Bukowski, W. M. (2014). Dehumanization in children: The link with moral disengagement in bullying and victimization. Aggressive Behavior, 40(4), 320-328. doi:10.1002/ab.21522

Viechtbauer, W. (2010). Conducting meta-analyses in R with the metafor Package. Journal of Statistical Software, 1(3), 1-48. doi:10.18637/jss.v036.i03

Walker, L. O.; Avant, K. C. (2018). Strategies for theory construction in nursing. New York: Pearson.

Wang, C. X.; Ryoo, J. H.; Swearer, S. M.; Turner, R.; Goldberg, T. S. (2017). Longitudinal relationships between bullying and moral disengagement among adolescents. Journal of Youth and Adolescence, 46(6), 1304-1317. doi:10.1007/ s10964-016-0577-0

Wang, X. C.; Yang, L.; Gao, L.; Yang, J. P.; Lei, L.; Wang, C. Y. (2017). Childhood maltreatment and Chinese adolescents' bullying and defending: The mediating role of moral disengagement. Child Abuse \& Neglect, 69, 134-144. doi:10.1016/j.chiabu.2017.04.016

Zequinão, M. A., Medeiros, P., Silva, J. L., Pereira, B. O., \& Cardoso, F. L. (2020). Sociometric status of participants involved in school bullying. Paidéia (Ribeirão Preto), 30, e3011. doi:10.1590/1982-4327e3011

O presente estudo foi realizado com apoio da Coordenação de Aperfeiçoamento de Pessoal de Nível Superior Brasil (CAPES) - Código de Financiamento 001 . O primeiro, penúltimo e último autores são pesquisadores bolsistas de Produtividade em Pesquisa do Conselho Nacional de Desenvolvimento Científico e Tecnológico - CNPq.

Recebido: 01 de maio de 2019 Aprovado: 08 de setembro de 2020 